\title{
Stability of solitary waves for derivative nonlinear Schrödinger equation
}

\author{
Mathieu Colin ${ }^{\mathrm{a}, *}$, Masahito Ohta ${ }^{\mathrm{b}}$ \\ a Mathématiques Appliquées de Bordeaux, Université Bordeaux I, 451 cours de la libération, 33405 Talence Cedex, France \\ ${ }^{\mathrm{b}}$ Department of Mathematics, Faculty of Science, Saitama University, Saitama 338-8570, Japan \\ Received 15 February 2005; received in revised form 7 September 2005; accepted 27 September 2005 \\ Available online 2 February 2006
}

\begin{abstract}
This paper is concerned with the so-called Derivative Nonlinear Schrödinger equation. This equation is known to have a two-parameter family of solitary waves solutions. We prove orbital stability of these particular solutions for the whole range of parameters values by using variational methods.

(C) 2006 L'Association Publications de l'Institut Henri Poincaré. Published by Elsevier B.V. All rights reserved
\end{abstract}

\section{Résumé}

Cet article concerne l'équation de Schrödinger Non Linéaire Dérivée. Cette équation possède une famille d'ondes solitaires dépendantes de deux paramètres. Nous prouvons la stabilité orbitale de ces solutions particulières pour toutes les valeurs des paramètres en utilisant des méthodes variationnelles.

(C) 2006 L'Association Publications de l'Institut Henri Poincaré. Published by Elsevier B.V. All rights reserved

MSC: 35A15; 35B35; 35Q55

Keywords: Solitary wave; Orbital stability; DNLS

\section{Introduction and main result}

In this paper, we study orbital stability of solitary wave solutions for the derivative nonlinear Schrödinger equation (DNLS):

$$
\mathrm{i} \partial_{t} u+\partial_{x}^{2} u+\mathrm{i} \partial_{x}\left(|u|^{2} u\right)=0, \quad(t, x) \in \mathbb{R} \times \mathbb{R} .
$$

Eq. (1.1) appears in plasma physics (see, e.g., [14,15]). It is known (see [9] and Section 2 below) that (1.1) has a two-parameter family of solitary wave solutions of the form:

$$
u_{\omega, c}(t, x)=\phi_{\omega, c}(x-c t) \exp \left\{\mathrm{i} \omega t+\mathrm{i} \frac{c}{2}(x-c t)-\frac{3}{4} \mathrm{i} \int_{-\infty}^{x-c t}\left|\phi_{\omega, c}(\eta)\right|^{2} \mathrm{~d} \eta\right\},
$$

\footnotetext{
* Corresponding author.

E-mail addresses: mcolin@math.u-bordeaux1.fr (M. Colin), mohta@ rimath.saitama-u.ac.jp (M. Ohta).
} 
where $(\omega, c) \in \mathbb{R}^{2}, c^{2}<4 \omega$, and

$$
\phi_{\omega, c}(x)=\left[\frac{\sqrt{\omega}}{4 \omega-c^{2}}\left\{\cosh \left(\sqrt{4 \omega-c^{2}} x\right)-\frac{c}{2 \sqrt{\omega}}\right\}\right]^{-1 / 2},
$$

which is a positive solution of

$$
-\partial_{x}^{2} \phi+\left(\omega-\frac{c^{2}}{4}\right) \phi+\frac{c}{2}|\phi|^{2} \phi-\frac{3}{16}|\phi|^{4} \phi=0, \quad x \in \mathbb{R} .
$$

In [9], Guo and Wu show that $u_{\omega, c}(t)$ is orbitally stable for DNLS (1.1) if $c<0$ and $c^{2}<4 \omega$. The case $c \geqslant 0$ is not studied in [9]. The proof in [9] is based on the general theory of Grillakis, Shatah and Strauss [7,8] and the spectral analysis of linearized operators. In [9], it is remarked that DNLS (1.1) cannot be written in a Hamiltonian form, so that the abstract theory of Grillakis, Shatah and Strauss [7,8] cannot be applied to (1.1) directly. Instead, the authors use an alternate stability theorem (see Theorem 2 in [9]), whose proof they omit.

In this paper, we shall prove that $u_{\omega, c}(t)$ is orbitally stable for DNLS (1.1) for any $(\omega, c) \in \mathbb{R}^{2}$ satisfying $c^{2}<4 \omega$, using the variational method related to the solitary waves (see Sections 3 and 4) as in Shatah [22] (see also [17]). We also use gauge transformations to rewrite (1.1) in a Hamiltonian form (see (2.5) below). The spectral analysis of linearized operators is not needed in our proof.

For later use, let us consider more general equation of the form:

$$
\mathrm{i} \partial_{t} u+\partial_{x}^{2} u+\mathrm{i} \lambda|u|^{2} \partial_{x} u+\mathrm{i} \mu u^{2} \partial_{x} \bar{u}+a|u|^{2} u+b|u|^{4} u=0,
$$

where $\lambda, \mu, a, b \in \mathbb{R}$. Recall that the Cauchy problem for (1.5) is locally well-posed in the energy space $H^{1}(\mathbb{R})$. It is proved by Ozawa [21] (see also [10-12]) that for any $u_{0} \in H^{1}(\mathbb{R})$ there exists a unique solution $u \in$ $C\left(\left[0, T_{\max }\left[, H^{1}(\mathbb{R})\right)\right.\right.$ of $(1.5)$ with initial data $u(0)=u_{0}$. Moreover, the solution $u(t)$ satisfies three conservation laws

$$
\mathcal{E}(u(t))=\mathcal{E}\left(u_{0}\right), \quad \mathcal{Q}(u(t))=\mathcal{Q}\left(u_{0}\right), \quad \mathcal{P}(u(t))=\mathcal{P}\left(u_{0}\right),
$$

for all $t \in\left[0, T_{\max }[\right.$, where

$$
\begin{aligned}
& \mathcal{E}(u)=\frac{1}{2}\left\|\partial_{x} u\right\|_{2}^{2}+\frac{\lambda+\mu}{4} \operatorname{Im} \int_{\mathbb{R}}|u|^{2} \bar{u} \partial_{x} u \mathrm{~d} x-\frac{a}{4}\|u\|_{4}^{4}-\left(b-\frac{5 \mu(\lambda+\mu)}{96}\right)\|u\|_{6}^{6}, \\
& \mathcal{Q}(u)=\frac{1}{2}\|u\|_{2}^{2}, \quad \mathcal{P}(u)=-\frac{1}{2} \operatorname{Im} \int_{\mathbb{R}} \bar{u} \partial_{x} u \mathrm{~d} x-\frac{\mu}{4}\|u\|_{4}^{4},
\end{aligned}
$$

and $\|\cdot\|_{p}$ denotes the $L^{p}(\mathbb{R})$ norm. For the well-posedness of the Cauchy problem for (1.1) in $H^{s}(\mathbb{R})$ with $s<1$, we refer to $[1,24,25]$.

Here, we give the definition of orbital stability.

Definition 1. Let $U=U(t, x)$ be a solitary wave solution of (1.5). We say that $U(t)$ is orbitally stable for (1.5) if for any $\varepsilon>0$ there exists $\delta>0$ such that if $u_{0} \in H^{1}(\mathbb{R})$ satisfies $\left\|u_{0}-U(0)\right\|_{H^{1}}<\delta$, then the solution $u(t)$ of (1.5) with initial data $u(0)=u_{0}$ exists globally in time and satisfies

$$
\sup _{t \geqslant 0} \inf _{(\theta, y) \in \mathbb{R}^{2}}\left\|u(t)-\mathrm{e}^{\mathrm{i} \theta} U(t, \cdot-y)\right\|_{H^{1}}<\varepsilon .
$$

Otherwise, $U(t)$ is said to be orbitally unstable.

Now we state the main result of this paper.

Theorem 1. For any $(\omega, c) \in \mathbb{R}^{2}$ satisfying $c^{2}<4 \omega$, the solitary wave solution $u_{\omega, c}(t)$ given by (1.2) is orbitally stable for DNLS (1.1). 
Remark 1. Note that $v_{\omega, c}(t, x)=\mathrm{e}^{\mathrm{i}\left(\omega-c^{2} / 4\right) t} \phi_{\omega, c}(x)$ is a solitary wave solution of the following nonlinear Schrödinger equation (NLS):

$$
\mathrm{i} \partial_{t} v+\partial_{x}^{2} v-\frac{c}{2}|v|^{2} v+\frac{3}{16}|v|^{4} v=0
$$

and it is known that $v_{\omega, c}(t)$ is orbitally stable for (1.7) if $c<0$, and it is orbitally unstable for (1.7) if $c \geqslant 0$ (see [18]). In fact, we have

$$
\left\|\phi_{\omega, c}\right\|_{2}^{2}=8 \tan ^{-1} \sqrt{\frac{2 \sqrt{\omega}+c}{2 \sqrt{\omega}-c}}
$$

for any $(\omega, c) \in \mathbb{R}^{2}$ satisfying $c^{2}<4 \omega$ (see Section 5). Thus, $\partial_{\omega}\left\|\phi_{\omega, c}\right\|_{2}^{2}$ is positive if $c<0$, and it is negative if $c>0$. The orbital stability and instability of $v_{\omega, c}(t)$ for NLS (1.7) follow from the variation of $\left\|\phi_{\omega, c}\right\|_{2}^{2}$ with respect to $\omega$ and the general theory of Grillakis, Shatah and Strauss [7,8] (see also [5,23,27]). For the case $c=0$, the strong instability of $v_{\omega, c}(t)$ for NLS (1.7) is proved by Weinstein [26]. Moreover, for the case $c>0$, we can prove the strong instability of $v_{\omega, c}(t)$ for NLS (1.7) in the same way as in [20] (see also [2,19]).

Remark 2. On one hand, it is known that if $u_{0} \in H^{1}(\mathbb{R})$ satisfies $\left\|u_{0}\right\|_{2}^{2}<\left\|\phi_{\omega, 0}\right\|_{2}^{2}=2 \pi$, then the solution $u(t)$ of DNLS (1.1) with initial data $u(0)=u_{0}$ exists globally in time (see [11,12,21]). On the other hand, little is known for the case $\left\|u_{0}\right\|_{2}^{2} \geqslant 2 \pi$. Especially, it is an open problem whether DNLS (1.1) has finite time blowup solutions for large data. Since $\left\|u_{\omega, c}(0)\right\|_{2}^{2}=\left\|\phi_{\omega, c}\right\|_{2}^{2}$, it follows from (1.8) that $\left\|u_{\omega, c}(0)\right\|_{2}^{2}<2 \pi$ if $c<0$, and $2 \pi \leqslant\left\|u_{\omega, c}(0)\right\|_{2}^{2}<4 \pi$ if $c \geqslant 0$. By Theorem $1, u_{\omega, c}(t)$ is orbitally stable for DNLS (1.1) even if $c \geqslant 0$, which gives some information about the global existence result for large initial data $\left\|u_{0}\right\|_{2}^{2} \geqslant 2 \pi$.

The plan of this paper is as follows. In Section 2, we state Theorem 3, which gives sufficient conditions for the orbital stability of a solitary wave of a simplified equation (2.4), and show that the conclusion of Theorem 1 follows from that of Theorem 3 via a gauge transformation. In Section 3, we give a variational characterization of solitary wave solutions. In Section 4, we give the proof of Theorem 3 using the variational characterization proved in Section 3 and the arguments in [22] and [17]. Finally, in Section 5, we use elementary computations to verify the conditions in the hypothesis of Theorem 3.

\section{Proof of Theorem 1}

In this section, we prove Theorem 1. First, we rewrite DNLS (1.1) in a Hamiltonian form by using a gauge transformation. For $v \in \mathbb{R}$, we define $G_{v}: H^{1}(\mathbb{R}) \rightarrow H^{1}(\mathbb{R})$ by

$$
G_{\nu}(u)(x)=u(x) \exp \left(v \mathrm{i} \int_{-\infty}^{x}|u(\eta)|^{2} \mathrm{~d} \eta\right), \quad u \in H^{1}(\mathbb{R}) .
$$

Note that $G_{v}^{-1}=G_{-v}$ and

$$
\mathrm{e}^{\mathrm{i} \theta} G_{v}(u)(x-y)=G_{v}\left(\mathrm{e}^{\mathrm{i} \theta} u(\cdot-y)\right)(x)
$$

for $u \in H^{1}(\mathbb{R}), x, y, \theta \in \mathbb{R}$. Moreover, there exists $C=C(v)>0$ such that

$$
\left\|G_{v}(u)-G_{v}(v)\right\|_{H^{1}} \leqslant C\left(1+\|u\|_{H^{1}}^{4}+\|v\|_{H^{1}}^{4}\right)\|u-v\|_{H^{1}}
$$

for $u, v \in H^{1}(\mathbb{R})$. By $v(t)=G_{v}(u(t))$, Eq. (1.5) is transformed to an equation of the same form with different coefficients:

$$
\mathrm{i} \partial_{t} v+\partial_{x}^{2} v+\mathrm{i} \tilde{\lambda}|v|^{2} \partial_{x} v+\mathrm{i} \tilde{\mu} v^{2} \partial_{x} \bar{v}+a|v|^{2} v+\tilde{b}|v|^{4} v=0,
$$

where

$$
\tilde{\lambda}=\lambda-2 v, \quad \tilde{\mu}=\mu-2 v, \quad \tilde{b}=b+v\left(v+\frac{\lambda}{2}-\frac{3}{2} \mu\right) .
$$


Note that to derive (2.3), we have used the following equality on $u$

$$
\partial_{t}|u|^{2}+\partial_{x}\left\{2 \operatorname{Im}\left(\bar{u} \partial_{x} u\right)+\frac{1}{2}(\lambda+\mu)|u|^{4}\right\}=0,
$$

which is obtained by a straightforward computation on Eq. (1.5). In particular, by $v(t)=G_{1 / 2}(u(t))$, DNLS (1.1) is transformed to

$$
\mathrm{i} \partial_{t} v+\partial_{x}^{2} v+\mathrm{i}|v|^{2} \partial_{x} v=0
$$

Then, (2.4) can be written in a Hamiltonian form:

$$
\partial_{t} v=-\mathrm{i} E^{\prime}(v)
$$

where

$$
E(v)=\frac{1}{2}\left\|\partial_{x} v\right\|_{2}^{2}+\frac{1}{4} \operatorname{Im} \int_{\mathbb{R}}|v|^{2} \bar{v} \partial_{x} v \mathrm{~d} x .
$$

Note that $E, Q$ and $P$ are conserved quantities of (2.4), where

$$
Q(v)=\frac{1}{2}\|v\|_{2}^{2}, \quad P(v)=-\frac{1}{2} \operatorname{Im} \int_{\mathbb{R}} \bar{v} \partial_{x} v \mathrm{~d} x
$$

(see (1.5) and (1.6)), and that

$$
E^{\prime}(v)=-\partial_{x}^{2} v-\mathrm{i}|v|^{2} \partial_{x} v, \quad Q^{\prime}(v)=v, \quad P^{\prime}(v)=\mathrm{i} \partial_{x} v .
$$

For a while, we consider Eq. (2.4) instead of (1.1). For $(\omega, c) \in \mathbb{R}^{2}$, we define the functional $S_{\omega, c}$ on $H^{1}(\mathbb{R})$ by

$$
\begin{aligned}
S_{\omega, c}(v) & =E(v)+\omega Q(v)+c P(v) \\
& =\frac{1}{2}\left\|\partial_{x} v\right\|_{2}^{2}+\frac{\omega}{2}\|v\|_{2}^{2}-\frac{c}{2} \operatorname{Im} \int_{\mathbb{R}} \bar{v} \partial_{x} v \mathrm{~d} x+\frac{1}{4} \operatorname{Im} \int_{\mathbb{R}}|v|^{2} \bar{v} \partial_{x} v \mathrm{~d} x .
\end{aligned}
$$

Let $\mathcal{G}_{\omega, c}$ be the set of all nontrivial critical points of $S_{\omega, c}$, i.e., $\varphi \in \mathcal{G}_{\omega, c}$ if and only if $\varphi \in H^{1}(\mathbb{R}) \backslash\{0\}$ satisfies

$$
-\partial_{x}^{2} \varphi-\mathrm{i}|\varphi|^{2} \partial_{x} \varphi+\omega \varphi+\mathrm{i} c \partial_{x} \varphi=0, \quad x \in \mathbb{R} .
$$

By (2.5), (2.8) and (2.9), we see that if $\varphi \in \mathcal{G}_{\omega, c}$ then $\mathrm{e}^{\mathrm{i} \omega t} \varphi(x-c t)$ is a solitary wave solution of (2.4). By the transformation

$$
\phi(x)=\varphi(x) \exp \left(-\frac{c}{2} \mathrm{i} x+\frac{\mathrm{i}}{4} \int_{-\infty}^{x}|\varphi(\eta)|^{2} \mathrm{~d} \eta\right),
$$

Eq. (2.10) is transformed to

$$
-\partial_{x}^{2} \phi+\left(\omega-\frac{c^{2}}{4}\right) \phi+\frac{1}{2} \operatorname{Im}\left(\bar{\phi} \partial_{x} \phi\right) \phi+\frac{c}{2}|\phi|^{2} \phi-\frac{3}{16}|\phi|^{4} \phi=0, \quad x \in \mathbb{R},
$$

and vice versa. When $c^{2}<4 \omega$, since $\phi_{\omega, c}$ given by (1.3) is a positive solution of (1.4), we have $\operatorname{Im}\left(\bar{\phi}_{\omega, c} \partial_{x} \phi_{\omega, c}\right)=0$ and $\phi_{\omega, c}$ satisfies (2.12). Therefore, $\varphi_{\omega, c}$ defined by

$$
\varphi_{\omega, c}(x)=\phi_{\omega, c}(x) \exp \left(\frac{c}{2} \mathrm{i} x-\frac{\mathrm{i}}{4} \int_{-\infty}^{x}\left|\phi_{\omega, c}(\eta)\right|^{2} \mathrm{~d} \eta\right)
$$

satisfies (2.10), i.e., $\varphi_{\omega, c} \in \mathcal{G}_{\omega, c}$ if $c^{2}<4 \omega$. The following lemma gives a characterization of the set $\mathcal{G}_{\omega, c}$.

Lemma 2. For any $(\omega, c) \in \mathbb{R}^{2}$ satisfying $c^{2}<4 \omega$, we have

$$
\mathcal{G}_{\omega, c}=\left\{\mathrm{e}^{\mathrm{i} \theta} \varphi_{\omega, c}(\cdot-y):(\theta, y) \in \mathbb{R}^{2}\right\} .
$$


Proof. Since it is trivial that $\left\{\mathrm{e}^{\mathrm{i} \theta} \varphi_{\omega, c}(\cdot-y):(\theta, y) \in \mathbb{R}^{2}\right\} \subset \mathcal{G}_{\omega, c}$, we have only to show the converse. Let $\varphi \in \mathcal{G}_{\omega, c}$. Then, $\varphi \in H^{1}(\mathbb{R}) \backslash\{0\}$ satisfies (2.10), and $\phi$ given by (2.11) satisfies (2.12). Let $f=\operatorname{Re} \phi$ and $g=\operatorname{Im} \phi$. Then, $f$ and $g$ satisfy $\partial_{x}^{2} f=A(\phi) f$ and $\partial_{x}^{2} g=A(\phi) g$, where we put

$$
A(\phi)=\omega-\frac{c^{2}}{4}+\frac{1}{2} \operatorname{Im}\left(\bar{\phi} \partial_{x} \phi\right)+\frac{c}{2}|\phi|^{2}-\frac{3}{16}|\phi|^{4} .
$$

Thus, we have $\partial_{x}\left(f \partial_{x} g-g \partial_{x} f\right)=0$ for any $x \in \mathbb{R}$. Since $f, g \in H^{1}(\mathbb{R})$, we see that $\operatorname{Im}\left(\bar{\phi} \partial_{x} \phi\right)=f \partial_{x} g-g \partial_{x} f=0$ for any $x \in \mathbb{R}$, so that $\phi$ satisfies (1.4). Therefore, there exists $(\theta, y) \in \mathbb{R}^{2}$ such that $\phi=\mathrm{e}^{\mathrm{i} \theta} \phi_{\omega, c}(\cdot-y)$ (see Cazenave [4, Theorem 8.1.6]). Finally, by (2.11) and (2.13), we conclude the lemma.

Next, we consider the orbital stability of solitary wave solution

$$
v_{\omega, c}(t, x)=\mathrm{e}^{\mathrm{i} \omega t} \varphi_{\omega, c}(x-c t)
$$

of (2.4). Note that the solitary wave solution $u_{\omega, c}(t)$ of DNLS (1.1) is given by $u_{\omega, c}(t)=G_{-1 / 2}\left(v_{\omega, c}(t)\right)$ (see (1.2)). For $(\omega, c) \in \mathbb{R}^{2}$ satisfying $c^{2}<4 \omega$, we define a function $d(\omega, c)$ by

$$
d(\omega, c)=S_{\omega, c}\left(\varphi_{\omega, c}\right) .
$$

Our sufficient condition for orbital stability is stated in terms of the derivatives of $d(\omega, c)$ as follows.

Theorem 3. Let $\left(\omega_{0}, c_{0}\right) \in \mathbb{R}^{2}$ satisfy $c_{0}^{2}<4 \omega_{0}$. Assume that there exists $\xi \in \mathbb{R}^{2}$ such that

$$
\left\langle d^{\prime}\left(\omega_{0}, c_{0}\right), \xi\right\rangle \neq 0, \quad\left\langle d^{\prime \prime}\left(\omega_{0}, c_{0}\right) \xi, \xi\right\rangle>0,
$$

where $\langle\cdot, \cdot\rangle$ denotes the inner product on $\mathbb{R}^{2}$. Then the solitary wave solution $v_{\omega_{0}, c_{0}}(t)$ given by (2.14) is orbitally stable for (2.4).

Note that $d^{\prime}(\omega, c)=\left(\partial_{\omega} d(\omega, c), \partial_{c} d(\omega, c)\right)$, and by $S_{\omega, c}^{\prime}\left(\varphi_{\omega, c}\right)=0$ we have

$$
\partial_{\omega} d(\omega, c)=Q\left(\varphi_{\omega, c}\right)>0, \quad \partial_{c} d(\omega, c)=P\left(\varphi_{\omega, c}\right) .
$$

Since $d^{\prime \prime}\left(\omega_{0}, c_{0}\right)$ is a $2 \times 2$ symmetric matrix, if $\operatorname{det}\left[d^{\prime \prime}\left(\omega_{0}, c_{0}\right)\right]<0$ then $d^{\prime \prime}\left(\omega_{0}, c_{0}\right)$ has one positive eigenvalue. In addition, since $d^{\prime}\left(\omega_{0}, c_{0}\right) \neq(0,0)$, we see that if $\operatorname{det}\left[d^{\prime \prime}\left(\omega_{0}, c_{0}\right)\right]<0$ then there exists $\xi \in \mathbb{R}^{2}$ satisfying (2.15). Now, if $\partial_{\omega}^{2} d\left(\omega_{0}, c_{0}\right)>0$, then $(2.15)$ is satisfied by taking $\xi=(1,0)$. Therefore, as a corollary of Theorem 3 , we have the following (compare with Theorem 2 in [9]).

Corollary 4. Let $\left(\omega_{0}, c_{0}\right) \in \mathbb{R}^{2}$ satisfy $c_{0}^{2}<4 \omega_{0}$. Assume $\operatorname{det}\left[d^{\prime \prime}\left(\omega_{0}, c_{0}\right)\right]<0$ or $\partial_{\omega}^{2} d\left(\omega_{0}, c_{0}\right)>0$. Then the solitary wave solution $v_{\omega_{0}, c_{0}}(t)$ is orbitally stable for (2.4).

Moreover, by elementary computations, we have the following.

Lemma 5. For any $(\omega, c) \in \mathbb{R}^{2}$ satisfying $c^{2}<4 \omega$, we have

$$
\begin{aligned}
& Q\left(\varphi_{\omega, c}\right)=4 \tan ^{-1} \sqrt{\frac{2 \sqrt{\omega}+c}{2 \sqrt{\omega}-c}}, \quad P\left(\varphi_{\omega, c}\right)=\sqrt{4 \omega-c^{2}}, \\
& \operatorname{det}\left[d^{\prime \prime}(\omega, c)\right]=-\frac{1}{\omega}<0 .
\end{aligned}
$$

We will prove Theorem 3 and Lemma 5 in Sections 4 and 5, respectively. Now, we prove Theorem 1 using Corollary 4 and Lemma 5.

Proof of Theorem 1. Let $(\omega, c) \in \mathbb{R}^{2}$ satisfy $c^{2}<4 \omega$. By (2.18) and Corollary 4, the solitary wave solution $v_{\omega, c}(t)$ is orbitally stable for (2.4). Thus, the orbital stability of the solitary wave solution $u_{\omega, c}(t)$ for DNLS (1.1) follows from the fact $u_{\omega, c}(t)=G_{-1 / 2}\left(v_{\omega, c}(t)\right)$ and the properties (2.1), (2.2) of the gauge transform $G_{v}$. 
Remark 3. In [9], for the case $c<0$, they show $\operatorname{det}\left[d^{\prime \prime}(\omega, c)\right]<0$ to prove the orbital stability of $u_{\omega, c}(t)$ for DNLS (1.1). However, for the case $c<0$, we have $\partial_{\omega}^{2} d(\omega, c)=\partial_{\omega} Q\left(\varphi_{\omega, c}\right)>0$. By Corollary 4, we do not need to compute $\operatorname{det}\left[d^{\prime \prime}(\omega, c)\right]$ to obtain the orbital stability of $u_{\omega, c}(t)$ for the case $c<0$. On the other hand, for the case $c \geqslant 0$, we have $\partial_{\omega}^{2} d(\omega, c) \leqslant 0$. Therefore, we need to compute $\operatorname{det}\left[d^{\prime \prime}(\omega, c)\right]$ to prove the orbital stability for the case $c \geqslant 0$.

\section{Variational characterization}

In this section, we give a variational characterization of solitary wave solution $v_{\omega, c}(t)$ defined by (2.14), which will be used in the proof of Theorem 3 in the next section.

For $(\omega, c) \in \mathbb{R}^{2}$ satisfying $c^{2}<4 \omega$, we consider the following minimization problem:

$$
\mu(\omega, c)=\inf \left\{S_{\omega, c}(u): u \in H^{1}(\mathbb{R}) \backslash\{0\}, K_{\omega, c}(u)=0\right\},
$$

where $S_{\omega, c}$ is defined by (2.9) and

$$
K_{\omega, c}(u)=\left\|\partial_{x} u\right\|_{2}^{2}+\omega\|u\|_{2}^{2}-c \operatorname{Im} \int_{\mathbb{R}} \bar{u} \partial_{x} u \mathrm{~d} x+\operatorname{Im} \int_{\mathbb{R}}|u|^{2} \bar{u} \partial_{x} u \mathrm{~d} x .
$$

For convenience, we put

$$
\begin{aligned}
& L_{\omega, c}(u)=\left\|\partial_{x} u\right\|_{2}^{2}+\omega\|u\|_{2}^{2}-c \operatorname{Im} \int_{\mathbb{R}} \bar{u} \partial_{x} u \mathrm{~d} x, \\
& N(u)=-\operatorname{Im} \int_{\mathbb{R}}|u|^{2} \bar{u} \partial_{x} u \mathrm{~d} x .
\end{aligned}
$$

Then, we can write

$$
S_{\omega, c}(u)=\frac{1}{2} L_{\omega, c}(u)-\frac{1}{4} N(u), \quad K_{\omega, c}(u)=L_{\omega, c}(u)-N(u) .
$$

Let $\mathcal{M}_{\omega, c}$ be the set of all minimizers for (3.1), i.e.,

$$
\mathcal{M}_{\omega, c}=\left\{\varphi \in H^{1}(\mathbb{R}) \backslash\{0\}: S_{\omega, c}(\varphi)=\mu(\omega, c), K_{\omega, c}(\varphi)=0\right\} .
$$

Recall that $\mathcal{G}_{\omega, c}=\left\{\varphi \in H^{1}(\mathbb{R}) \backslash\{0\}: S_{\omega, c}^{\prime}(\varphi)=0\right\}$, and note that $K_{\omega, c}(u)=\left.\partial_{\lambda} S_{\omega, c}(\lambda u)\right|_{\lambda=1}$ for $u \in H^{1}(\mathbb{R})$. Thus, if $\varphi \in \mathcal{G}_{\omega, c}$, then we have $K_{\omega, c}(\varphi)=0$.

The main result in this section is as follows.

Proposition 6. Let $(\omega, c) \in \mathbb{R}^{2}$ satisfy $c^{2}<4 \omega$. If a sequence $\left\{u_{n}\right\} \subset H^{1}(\mathbb{R})$ satisfies

$$
S_{\omega, c}\left(u_{n}\right) \rightarrow \mu(\omega, c), \quad K_{\omega, c}\left(u_{n}\right) \rightarrow 0,
$$

then there exist a sequence $\left\{y_{n}\right\} \subset \mathbb{R}$ and $v \in \mathcal{M}_{\omega, c}$ such that $\left\{u_{n}\left(\cdot-y_{n}\right)\right\}$ has a subsequence that converges to $v$ strongly in $H^{1}(\mathbb{R})$.

To prove Proposition 6, we first prove a simple lemma.

Lemma 7. Let $(\omega, c) \in \mathbb{R}^{2}$ satisfy $c^{2}<4 \omega$.

(1) There exists a constant $C_{1}=C_{1}(\omega, c)>0$ such that

$$
L_{\omega, c}(u) \geqslant C_{1}\|u\|_{H^{1}}^{2}, \quad u \in H^{1}(\mathbb{R}) .
$$

(2) $\mu(\omega, c)>0$.

(3) If $u \in H^{1}(\mathbb{R})$ satisfies $K_{\omega, c}(u)<0$, then $L_{\omega, c}(u)>4 \mu(\omega, c)$. 
Proof. First, we show (1). For $\alpha>0$ and $u \in H^{1}(\mathbb{R})$, we have

$$
L_{\omega, c}(u)=(1-\alpha)\left\|\partial_{x} u\right\|_{2}^{2}+\frac{1}{\alpha}\left\|\alpha \partial_{x} u-\frac{c}{2} \mathrm{i} u\right\|_{2}^{2}+\left(\omega-\frac{c^{2}}{4 \alpha}\right)\|u\|_{2}^{2} .
$$

Since $c^{2}<4 \omega$, taking $\left.\alpha \in\right] c^{2} / 4 \omega, 1\left[\right.$, we have (1). Next, we show (2). Let $u$ be any element of $H^{1}(\mathbb{R}) \backslash\{0\}$ satisfying $K_{\omega, c}(u)=0$. By (1) and the Sobolev inequality, there exists a constant $C_{2}>0$ such that

$$
C_{1}\|u\|_{H^{1}}^{2} \leqslant L_{\omega, c}(u)=N(u) \leqslant C_{2}\|u\|_{H^{1}}^{4} .
$$

Since $u \neq 0$, we have $\|u\|_{H^{1}}^{2} \geqslant C_{1} / C_{2}$. Thus, by (3.1), (3.5) and (3.7), we see that

$$
\mu(\omega, c)=\frac{1}{4} \inf \left\{L_{\omega, c}(u): u \in H^{1}(\mathbb{R}) \backslash\{0\}, K_{\omega, c}(u)=0\right\} \geqslant \frac{C_{1}^{2}}{4 C_{2}}>0 .
$$

Finally, we show (3). Let $u \in H^{1}(\mathbb{R})$ satisfy $K_{\omega, c}(u)<0$. Then, there exists $\left.\lambda_{1} \in\right] 0,1\left[\right.$ such that $K_{\omega, c}\left(\lambda_{1} u\right)=$ $\lambda_{1}^{2} L_{\omega, c}(u)-\lambda_{1}^{4} N(u)=0$. Since $u \neq 0$, by (3.9), we have $4 \mu(\omega, c) \leqslant L_{\omega, c}\left(\lambda_{1} u\right)=\lambda_{1}^{2} L_{\omega, c}(u)<L_{\omega, c}(u)$.

To prove Proposition 6, we use the following compactness lemmas due to Fröhlich, Lieb and Loss [6], Lieb [13] and Brézis and Lieb [3].

Lemma 8. Let $\left\{f_{n}\right\}$ be a bounded sequence in $H^{1}(\mathbb{R})$. Assume that there exists $\left.p \in\right] 2, \infty[$ such that

$$
\limsup _{n \rightarrow \infty}\left\|f_{n}\right\|_{p}>0 \text {. }
$$

Then, there exist $\left\{y_{n}\right\} \subset \mathbb{R}$ and $f \in H^{1}(\mathbb{R}) \backslash\{0\}$ such that $\left\{f_{n}\left(\cdot-y_{n}\right)\right\}$ has a subsequence that converges to $f$ weakly in $H^{1}(\mathbb{R})$.

For the proof of Lemma 8, see [6] and [13].

Lemma 9. Let $2 \leqslant p<\infty$ and $\left\{f_{n}\right\}$ be a bounded sequence in $L^{p}(\mathbb{R})$. Assume that $f_{n} \rightarrow f$ a.e. in $\mathbb{R}$. Then we have

$$
\left\|f_{n}\right\|_{p}^{p}-\left\|f_{n}-f\right\|_{p}^{p}-\|f\|_{p}^{p} \rightarrow 0 .
$$

For the proof of Lemma 9, see [3].

Now, using Lemmas 8 and 9, we prove Proposition 6. Similar arguments can be found in [16] and [19].

Proof of Proposition 6. In what follows, we shall often extract subsequences without mentioning this fact explicitly.

Step 1. By (3.5) and (3.6), we have $L_{\omega, c}\left(u_{n}\right)=4 S_{\omega, c}\left(u_{n}\right)-K_{\omega, c}\left(u_{n}\right) \rightarrow 4 \mu(\omega, c)$. Thus, by (3.7), we see that $\left\{u_{n}\right\}$ is bounded in $H^{1}(\mathbb{R})$.

Step 2. We show $\limsup _{n \rightarrow \infty}\left\|u_{n}\right\|_{6}>0$ by contradiction. Suppose that $\lim _{n \rightarrow \infty}\left\|u_{n}\right\|_{6}=0$. Then, by the Cauchy-Schwarz inequality and the boundedness of $\left\{u_{n}\right\}$ in $H^{1}(\mathbb{R})$, we have $\left|N\left(u_{n}\right)\right| \leqslant\left\|u_{n}\right\|_{6}^{3}\left\|\partial_{x} u\right\|_{2} \rightarrow 0$. Since $K_{\omega, c}\left(u_{n}\right) \rightarrow 0$, we have $L_{\omega, c}\left(u_{n}\right) \rightarrow 0$. Moreover, by Lemma $7(2)$, we have $L_{\omega, c}\left(u_{n}\right) \rightarrow 4 \mu(\omega, c)>0$. This is a contradiction.

Step 3. By Steps 1 and 2 and Lemma 8, there exist $\left\{y_{n}\right\} \subset \mathbb{R}$, a subsequence of $\left\{u_{n}\left(\cdot-y_{n}\right)\right\}$ (we denote it by $\left\{v_{n}\right\}$ ) and $v \in H^{1}(\mathbb{R}) \backslash\{0\}$ such that $v_{n} \rightarrow v$ weakly in $H^{1}(\mathbb{R})$.

Step 4. We first note that

$$
K_{\omega, c}(u)=\left\|\partial_{x} u-\frac{c}{2} \mathrm{i} u+\frac{\mathrm{i}}{2}|u|^{2} u\right\|_{2}^{2}+\left(\omega-\frac{c^{2}}{4}\right)\|u\|_{2}^{2}+\frac{c}{2}\|u\|_{4}^{4}-\frac{1}{4}\|u\|_{6}^{6}
$$

for any $u \in H^{1}(\mathbb{R})$. Since $\left\{v_{n}\right\}$ is bounded in $H^{1}(\mathbb{R})$ and $v_{n} \rightarrow v$ a.e. in $\mathbb{R}$, by Lemma 9 , we have $\left\|v_{n}\right\|_{p}^{p}-\| v_{n}-$ $v\left\|_{p}^{p}-\right\| v \|_{p}^{p} \rightarrow 0$ for $2 \leqslant p<\infty$. Moreover, if we put

$$
w_{n}=\partial_{x} v_{n}-\frac{c}{2} \mathrm{i} v_{n}+\frac{\mathrm{i}}{2}\left|v_{n}\right|^{2} v_{n}, \quad w=\partial_{x} v-\frac{c}{2} \mathrm{i} v+\frac{\mathrm{i}}{2}|v|^{2} v
$$


then $w_{n} \rightarrow w$ weakly in $L^{2}(\mathbb{R})$. Thus, we have $\left\|w_{n}\right\|_{2}^{2}-\left\|w_{n}-w\right\|_{2}^{2}-\|w\|_{2}^{2} \rightarrow 0$. Therefore, by (3.10), we have

$$
K_{\omega, c}\left(v_{n}\right)-K_{\omega, c}\left(v_{n}-v\right)-K_{\omega, c}(v) \rightarrow 0 .
$$

Similarly, by (3.8) with $c^{2} /(4 \omega)<\alpha<1$, we have

$$
L_{\omega, c}\left(v_{n}\right)-L_{\omega, c}\left(v_{n}-v\right)-L_{\omega, c}(v) \rightarrow 0 .
$$

Step 5. We show $K_{\omega, c}(v) \leqslant 0$ by contradiction. Suppose that $K_{\omega, c}(v)>0$. By (3.6) and (3.11), we have

$$
K_{\omega, c}\left(v_{n}-v\right) \rightarrow-K_{\omega, c}(v)<0,
$$

which shows $K_{\omega, c}\left(v_{n}-v\right)<0$ for large $n$. By Lemma 7(3), we have $L_{\omega, c}\left(v_{n}-v\right) \geqslant 4 \mu(\omega, c)$ for large $n$. Since $L_{\omega, c}\left(v_{n}\right) \rightarrow 4 \mu(\omega, c)$, by (3.12), we have

$$
L_{\omega, c}(v)=\lim _{n \rightarrow \infty}\left\{L_{\omega, c}\left(v_{n}\right)-L_{\omega, c}\left(v_{n}-v\right)\right\} \leqslant 0 .
$$

Moreover, by Step 3 and Lemma 7(1), we have $v \neq 0$ and $L_{\omega, c}(v)>0$. This is a contradiction. Hence, we have $K_{\omega, c}(v) \leqslant 0$.

Step 6. By (3.9), Lemma 7(3) and the weakly lower semicontinuity of $L_{\omega, c}$, we have

$$
4 \mu(\omega, c) \leqslant L_{\omega, c}(v) \leqslant \liminf _{n \rightarrow \infty} L_{\omega, c}\left(v_{n}\right)=4 \mu(\omega, c) .
$$

Thus, we have $L_{\omega, c}(v)=4 \mu(\omega, c)(v)$ and $v_{n} \rightarrow v$ strongly in $H^{1}(\mathbb{R})$. Moreover, by Step 5, (3.5) and Lemma 7(3), we have $K_{\omega, c}(v)=0$ and $S_{\omega, c}(v)=\mu(\omega, c)$, which show $v \in \mathcal{M}_{\omega, c}$. This completes the proof.

Finally, we prove the following lemma, which gives a characterization of the set $\mathcal{M}_{\omega, c}$.

Lemma 10. Let $(\omega, c) \in \mathbb{R}^{2}$ satisfy $c^{2}<4 \omega$. Then, we have $\mathcal{M}_{\omega, c}=\mathcal{G}_{\omega, c}$. In particular, we have $d(\omega, c)=\mu(\omega, c)$.

Proof. First, we show that $\mathcal{M}_{\omega, c} \subset \mathcal{G}_{\omega, c}$. Let $\varphi \in \mathcal{M}_{\omega, c}$. Then, there exists a Lagrange multiplier $\lambda \in \mathbb{R}$ such that $S_{\omega, c}^{\prime}(\varphi)=\lambda K_{\omega, c}^{\prime}(\varphi)$. Thus, we have

$$
0=K_{\omega, c}(\varphi)=\left\langle S_{\omega, c}^{\prime}(\varphi), \varphi\right\rangle=\lambda\left\langle K_{\omega, c}^{\prime}(\varphi), \varphi\right\rangle .
$$

By $K_{\omega, c}(\varphi)=0,(3.7)$ and $\varphi \neq 0$, we have

$$
\left\langle K_{\omega, c}^{\prime}(\varphi), \varphi\right\rangle=2 L_{\omega, c}(\varphi)-4 N(\varphi)=-2 L_{\omega, c}(\varphi)<0 .
$$

Thus, we see that $\lambda=0$ and $\varphi \in \mathcal{G}_{\omega, c}$, which implies $\mathcal{M}_{\omega, c} \subset \mathcal{G}_{\omega, c}$. We now show the converse. Let $\varphi \in \mathcal{M}_{\omega, c}$. Then $\varphi \in \mathcal{G}_{\omega, c}$ and by Lemma 2 there exists $(\theta, y) \in \mathbb{R}^{2}$ such that $\varphi(\cdot)=\mathrm{e}^{\mathrm{i} \theta} \varphi_{\omega, c}(\cdot-y)$. It follows

$$
S_{\omega, c}(\varphi)=\mu(\omega, c)=S_{\omega, c}\left(\mathrm{e}^{\mathrm{i} \theta} \varphi_{\omega, c}(\cdot-y)\right)=S_{\omega, c}\left(\varphi_{\omega, c}\right),
$$

which implies $\varphi_{\omega, c} \in \mathcal{M}_{\omega, c}$. Finally, we conclude the proof by using Lemma 2.

\section{Proof of Theorem 3}

In this section, we prove Theorem 3 . The proof is based on the variational characterization of solitary wave solutions in Section 3 and the arguments in [22] and [17]. Throughout this section, we assume that $(\omega, c) \in \mathbb{R}^{2}$ satisfies $c^{2}<4 \omega$. We put

$$
\begin{aligned}
& \mathcal{A}_{\omega, c}^{+}=\left\{v \in H^{1}(\mathbb{R}) \backslash\{0\}: S_{\omega, c}(v)<d(\omega, c), K_{\omega, c}(v)>0\right\}, \\
& \mathcal{A}_{\omega, c}^{-}=\left\{v \in H^{1}(\mathbb{R}) \backslash\{0\}: S_{\omega, c}(v)<d(\omega, c), K_{\omega, c}(v)<0\right\}, \\
& \mathcal{B}_{\omega, c}^{+}=\left\{v \in H^{1}(\mathbb{R}) \backslash\{0\}: S_{\omega, c}(v)<d(\omega, c), N(v)<4 d(\omega, c)\right\}, \\
& \mathcal{B}_{\omega, c}^{-}=\left\{v \in H^{1}(\mathbb{R}) \backslash\{0\}: S_{\omega, c}(v)<d(\omega, c), N(v)>4 d(\omega, c)\right\},
\end{aligned}
$$

where $S_{\omega, c}, K_{\omega, c}$ and $N$ are defined by (2.9), (3.2) and (3.4), respectively, and note that $d(\omega, c)=\mu(\omega, c)$ by Lemma 10. 
Lemma 11. Let $(\omega, c) \in \mathbb{R}^{2}$ satisfy $c^{2}<4 \omega$.

(1) The sets $\mathcal{A}_{\omega, c}^{+}$and $\mathcal{A}_{\omega, c}^{-}$are invariant under the flow of (2.4), i.e., if $u_{0}$ belongs to $\mathcal{A}_{\omega, c}^{+}$(resp. $\mathcal{A}_{\omega, c}^{-}$), then the solution $u(t)$ of $(2.4)$ with $u(0)=u_{0}$ belongs to $\mathcal{A}_{\omega, c}^{+}\left(\right.$resp. $\left.\mathcal{A}_{\omega, c}^{-}\right)$as long as $u(t)$ exists.

(2) $\mathcal{A}_{\omega, c}^{+}=\mathcal{B}_{\omega, c}^{+}$and $\mathcal{A}_{\omega, c}^{-}=\mathcal{B}_{\omega, c}^{-}$.

Proof. (1) Let $u_{0} \in \mathcal{A}_{\omega, c}^{+}$and $T_{\max }$ be the maximal existence time of the solution $u(t)$ of (2.4) with $u(0)=u_{0}$. By $u_{0} \neq 0$ and the conservation laws (1.6), we have $u(t) \neq 0$ for $t \in\left[0, T_{\max }[\right.$. Since $E, Q, P$ defined by (2.6) and (2.7) are conserved quantities of (2.4), $S_{\omega, c}$ is also conserved. Thus, we have $S_{\omega, c}(u(t))=S_{\omega, c}\left(u_{0}\right)<d(\omega, c)$ for $t \in\left[0, T_{\max }\left[\right.\right.$. Moreover, by (3.1) and $\mu(\omega, c)=d(\omega, c)$, we see that $K_{\omega, c}(u(t)) \neq 0$ for $t \in\left[0, T_{\max }[\right.$. Since the function $t \mapsto K_{\omega, c}(u(t))$ is continuous, we have $K_{\omega, c}(u(t))>0$ for $t \in\left[0, T_{\max }\left[\right.\right.$. Hence, $\mathcal{A}_{\omega, c}^{+}$is invariant under the flow of (2.4). By the same way, we see that $\mathcal{A}_{\omega, c}^{-}$is invariant under the flow of (2.4).

(2) If $v \in \mathcal{A}_{\omega, c}^{+}$, then by (3.5) we have $N(v)=4 S_{\omega, c}(v)-2 K_{\omega, c}(v)<4 d(\omega, c)$, which shows $v \in \mathcal{B}_{\omega, c}^{+}$and $\mathcal{A}_{\omega, c}^{+} \subset \mathcal{B}_{\omega, c}^{+}$. Now, let $v \in \mathcal{B}_{\omega, c}^{+}$. We show $K_{\omega, c}(v)>0$ by contradiction. Suppose that $K_{\omega, c}(v) \leqslant 0$. Then, by (3.9) and Lemma 7(3), we have $L_{\omega, c}(v) \geqslant 4 \mu(\omega, c)=4 d(\omega, c)$. Thus, by (3.5), we have

$$
S_{\omega, c}(v)=\frac{1}{2} L_{\omega, c}(v)-\frac{1}{4} N(v) \geqslant d(\omega, c),
$$

which contradicts $S_{\omega, c}(v)<d(\omega, c)$. Therefore, we have $K_{\omega, c}(v)>0$, which shows $v \in \mathcal{A}_{\omega, c}^{+}$and $\mathcal{B}_{\omega, c}^{+} \subset \mathcal{A}_{\omega, c}^{+}$. Next, if $v \in \mathcal{A}_{\omega, c}^{-}$, then by Lemma 7(3) we have $L_{\omega, c}(v)>4 \mu(\omega, c)=4 d(\omega, c)$. Thus, by (3.5), we have $N(v)=$ $L_{\omega, c}(v)-K_{\omega, c}(v)>4 d(\omega, c)$, which shows $v \in \mathcal{B}_{\omega, c}^{-}$and $\mathcal{A}_{\omega, c}^{-} \subset \mathcal{B}_{\omega, c}^{-}$. Finally, if $v \in \mathcal{B}_{\omega, c}^{-}$, then by (3.5) we have $2 K_{\omega, c}(v)=4 S_{\omega, c}(v)-N(v)<4 d(\omega, c)-4 d(\omega, c)=0$, which shows $v \in \mathcal{A}_{\omega, c}^{-}$and $\mathcal{B}_{\omega, c}^{-} \subset \mathcal{A}_{\omega, c}^{-}$. This completes the proof.

Let $\left(\omega_{0}, c_{0}\right) \in \mathbb{R}^{2}$ satisfy $c_{0}^{2}<4 \omega_{0}$, and assume that there exists $\xi \in \mathbb{R}^{2}$ that satisfies (2.15). We define the function $h:]-\varepsilon_{0}, \varepsilon_{0}[\rightarrow \mathbb{R}$ by

$$
\left.h(\tau)=d\left(\left(\omega_{0}, c_{0}\right)+\tau \xi\right), \quad \tau \in\right]-\varepsilon_{0}, \varepsilon_{0}[
$$

for sufficiently small $\varepsilon_{0}>0$. Then, by (2.15), we have

$$
h^{\prime}(0)=\left\langle d^{\prime}\left(\omega_{0}, c_{0}\right), \xi\right\rangle \neq 0, \quad h^{\prime \prime}(0)=\left\langle d^{\prime \prime}\left(\omega_{0}, c_{0}\right) \xi, \xi\right\rangle>0 .
$$

Without loss of generality, we can assume that $h^{\prime}(0)>0$ by replacing $\xi$ by $-\xi$ if necessary. Moreover, by the continuity of $h^{\prime}$ and $h^{\prime \prime}$, we can choose $\varepsilon_{0}$ such that $h$ satisfies

$$
\left.h^{\prime}(\tau)>0, \quad h^{\prime \prime}(\tau)>0 \quad \text { for } \tau \in\right]-\varepsilon_{0}, \varepsilon_{0}[.
$$

Lemma 12. Let $\left(\omega_{0}, c_{0}\right) \in \mathbb{R}^{2}$ satisfy $c_{0}^{2}<4 \omega_{0}$. Assume that there exists $\xi \in \mathbb{R}^{2}$ that satisfies (2.15), and let $h$ be the increasing function defined by (4.1). Then, for any $\varepsilon \in] 0, \varepsilon_{0}\left[\right.$, there exists $\delta>0$ such that if $u_{0} \in H^{1}(\mathbb{R})$ satisfies $\left\|u_{0}-\varphi_{\omega_{0}, c_{0}}\right\|_{H^{1}}<\delta$, then the solution $u(t)$ of (2.4) with $u(0)=u_{0}$ satisfies $4 h(-\varepsilon)<N(u(t))<4 h(\varepsilon)$ for all $t \in\left[0, T_{\max }[\right.$.

Proof. As mentioned above, we can assume that $h$ satisfies (4.3). Let $\varepsilon \in] 0, \varepsilon_{0}$ [. Since $h$ is increasing, we have $h(-\varepsilon)<h(0)<h(\varepsilon)$. Moreover, by $K_{\omega_{0}, c_{0}}\left(\varphi_{\omega_{0}, c_{0}}\right)=0$ and $(3.5)$, we deduce $4 h(0)=4 d\left(\omega_{0}, c_{0}\right)=4 S_{\omega_{0}, c_{0}}\left(\varphi_{\omega_{0}, c_{0}}\right)=$ $N\left(\varphi_{\omega_{0}, c_{0}}\right)$. Thus, if $u_{0} \in H^{1}(\mathbb{R})$ satisfies $\left\|u_{0}-\varphi_{\omega_{0}, c_{0}}\right\|_{H^{1}}<\delta$ then we have $4 h(0)=N\left(u_{0}\right)+O(\delta)$, and $4 h(-\varepsilon)<$ $N\left(u_{0}\right)<4 h(\varepsilon)$ for sufficiently small $\delta>0$. Since $h( \pm \varepsilon)=d\left(\left(\omega_{0}, c_{0}\right) \pm \varepsilon \xi\right)$ and the sets $\mathcal{B}_{\omega_{0}, c_{0}}^{ \pm}$are invariant under the flow of (2.4) by Lemma 11, to conclude the proof, we have only to show that there exists $\delta>0$ such that if $u_{0} \in H^{1}(\mathbb{R})$ satisfies $\left\|u_{0}-\varphi_{\omega_{0}, c_{0}}\right\|_{H^{1}}<\delta$ then $S_{\left(\omega_{0}, c_{0}\right) \pm \varepsilon \xi}\left(u_{0}\right)<h( \pm \varepsilon)$. Assume that $u_{0} \in H^{1}(\mathbb{R})$ satisfies $\left\|u_{0}-\varphi_{\omega_{0}, c_{0}}\right\|_{H^{1}}<\delta$, and put $\xi=\left(\xi_{1}, \xi_{2}\right)$. Then, by (2.7), (2.9), (2.16) and (4.2), we have

$$
\begin{aligned}
S_{\left(\omega_{0}, c_{0}\right) \pm \varepsilon \xi}\left(u_{0}\right) & =S_{\left(\omega_{0}, c_{0}\right) \pm \varepsilon \xi}\left(\varphi_{\omega_{0}, c_{0}}\right)+\mathrm{O}(\delta) \\
& =S_{\omega_{0}, c_{0}}\left(\varphi_{\omega_{0}, c_{0}}\right) \pm \varepsilon\left\{\xi_{1} Q\left(\varphi_{\omega_{0}, c_{0}}\right)+\xi_{2} P\left(\varphi_{\omega_{0}, c_{0}}\right)\right\}+\mathrm{O}(\delta) \\
& =h(0) \pm \varepsilon h^{\prime}(0)+\mathrm{O}(\delta) .
\end{aligned}
$$


On the other hand, by the Taylor expansion, there exists $\left.\tau_{1}=\tau_{1}(\varepsilon) \in\right]-\varepsilon_{0}, \varepsilon_{0}[$ such that

$$
h( \pm \varepsilon)=h(0) \pm \varepsilon h^{\prime}(0)+\frac{\varepsilon^{2}}{2} h^{\prime \prime}\left(\tau_{1}\right) .
$$

Since $h^{\prime \prime}\left(\tau_{1}\right)>0$ by (4.3), we see that there exists $\delta>0$ such that if $u_{0} \in H^{1}(\mathbb{R})$ satisfies $\left\|u_{0}-\varphi_{\omega_{0}, c_{0}}\right\|_{H^{1}}<\delta$ then $S_{\left(\omega_{0}, c_{0}\right) \pm \varepsilon \xi}\left(u_{0}\right)<h( \pm \varepsilon)$. This completes the proof.

Now, we prove Theorem 3.

Proof of Theorem 3. We prove by contradiction. Suppose that $v_{\omega_{0}, c_{0}}(t)$ is not orbitally stable for (2.4). Then, there exist a constant $\varepsilon_{1}>0$, a sequence of solutions $\left\{u_{n}\right\}$ to (2.4), and a sequence $\left\{t_{n}\right\}$ in $] 0, \infty[$ such that

$$
\begin{aligned}
& u_{n}(0) \rightarrow \varphi_{\omega_{0}, c_{0}} \quad \text { in } H^{1}(\mathbb{R}), \\
& \inf _{(\theta, y) \in \mathbb{R}^{2}}\left\|u_{n}\left(t_{n}\right)-\mathrm{e}^{\mathrm{i} \theta} \varphi_{\omega_{0}, c_{0}}(\cdot-y)\right\|_{H^{1}} \geqslant \varepsilon_{1} .
\end{aligned}
$$

Then, since $E, Q$ and $P$ are conserved, by (2.9) and (4.4), we have

$$
S_{\omega_{0}, c_{0}}\left(u_{n}\left(t_{n}\right)\right)=S_{\omega_{0}, c_{0}}\left(u_{n}(0)\right) \rightarrow S_{\omega_{0}, c_{0}}\left(\varphi_{\omega_{0}, c_{0}}\right)=d\left(\omega_{0}, c_{0}\right) .
$$

Note that $d\left(\omega_{0}, c_{0}\right)=\mu\left(\omega_{0}, c_{0}\right)$ by Lemma 10. Moreover, by Lemma 12 and (4.4), we have

$$
N\left(u_{n}\left(t_{n}\right)\right) \rightarrow 4 d\left(\omega_{0}, c_{0}\right) .
$$

Thus, by (3.5), (4.6) and (4.7), we have

$$
K_{\omega_{0}, c_{0}}\left(u_{n}\left(t_{n}\right)\right)=2 S_{\omega_{0}, c_{0}}\left(u_{n}\left(t_{n}\right)\right)-\frac{1}{2} N\left(u_{n}\left(t_{n}\right)\right) \rightarrow 0 .
$$

Therefore, by (4.6), (4.8) and Proposition 6, there exist a sequence $\left\{y_{n}\right\} \subset \mathbb{R}$ and $v \in \mathcal{M}_{\omega_{0}, c_{0}}$ such that $\left\{u_{n}\left(t_{n}, \cdot-y_{n}\right)\right\}$ has a subsequence (we denote it by the same letter) that converges to $v$ in $H^{1}(\mathbb{R})$. Thus, by Lemmas 2 and 10 , we have

$$
\inf _{(\theta, y) \in \mathbb{R}^{2}}\left\|u_{n}\left(t_{n}\right)-\mathrm{e}^{\mathrm{i} \theta} \varphi_{\omega_{0}, c_{0}}(\cdot-y)\right\|_{H^{1}} \rightarrow 0,
$$

which contradicts (4.5). Hence, $v_{\omega_{0}, c_{0}}(t)$ is orbitally stable for (2.4).

\section{Proof of Lemma 5}

In this section, we prove Lemma 5 using the expression (1.3) of $\phi_{\omega, c}$ and elementary computations. In what follows, we put

$$
\alpha=4 \omega-c^{2}, \quad \beta=\frac{c}{2 \sqrt{\omega}} .
$$

Note that $\alpha>0$ and $-1<\beta<1$ by $c^{2}<4 \omega$. Then, by (1.3), we have

$$
\phi_{\omega, c}(x)^{2}=\frac{\alpha}{\sqrt{\omega}\{\cosh (\sqrt{\alpha} x)-\beta\}} .
$$

By (2.13) and by elementary formulas

$$
\begin{aligned}
& \int_{0}^{\infty} \frac{\mathrm{d} y}{\cosh 2 y-\beta}=\frac{1}{\sqrt{1-\beta^{2}}} \tan ^{-1} \sqrt{\frac{1+\beta}{1-\beta}}, \\
& \int_{0}^{\infty} \frac{\mathrm{d} y}{\{\cosh 2 y-\beta\}^{2}}=\frac{\beta}{\left(1-\beta^{2}\right)^{3 / 2}} \tan ^{-1} \sqrt{\frac{1+\beta}{1-\beta}}+\frac{1}{2\left(1-\beta^{2}\right)},
\end{aligned}
$$

we have 


$$
\begin{aligned}
& Q\left(\varphi_{\omega, c}\right)=\int_{0}^{\infty} \phi_{\omega, c}(x)^{2} \mathrm{~d} x=4 \tan ^{-1} \sqrt{\frac{1+\beta}{1-\beta}}, \\
& \int_{0}^{\infty} \phi_{\omega, c}(x)^{4} \mathrm{~d} x=8 c \tan ^{-1} \sqrt{\frac{1+\beta}{1-\beta}}+4 \sqrt{\alpha}, \\
& P\left(\varphi_{\omega, c}\right)=-\frac{c}{2} \int_{0}^{\infty} \phi_{\omega, c}(x)^{2} \mathrm{~d} x+\frac{1}{4} \int_{0}^{\infty} \phi_{\omega, c}(x)^{4} \mathrm{~d} x=\sqrt{\alpha},
\end{aligned}
$$

which imply (2.17). Moreover, by (2.17), we have

$$
\begin{aligned}
& \partial_{c} Q\left(\varphi_{\omega, c}\right)=\partial_{\omega} P\left(\varphi_{\omega, c}\right)=\frac{2}{\sqrt{4 \omega-c^{2}}}, \\
& \partial_{c} P\left(\varphi_{\omega, c}\right)=-\frac{c}{\sqrt{4 \omega-c^{2}}}, \quad \partial_{\omega} Q\left(\varphi_{\omega, c}\right)=-\frac{c}{\omega \sqrt{4 \omega-c^{2}}} .
\end{aligned}
$$

Thus, we have

$$
\operatorname{det}\left[d^{\prime \prime}(\omega, c)\right]=\partial_{\omega} Q\left(\varphi_{\omega, c}\right) \partial_{c} P\left(\varphi_{\omega, c}\right)-\partial_{c} Q\left(\varphi_{\omega, c}\right) \partial_{\omega} P\left(\varphi_{\omega, c}\right)=-\frac{1}{\omega},
$$

which shows (2.18). This completes the proof of Lemma 5.

\section{Acknowledgements}

Part of this work was done while M. Ohta was a visitor of Université Bordeaux I.M. Ohta wishes to thank Mathématiques Appliquées de Bordeaux for its hospitality. We also want to thank the referees for useful remarks and comments.

\section{References}

[1] H.A. Biagioni, F. Linares, Ill-posedness for the derivative Schrödinger and generalized Benjamin-Ono equations, Trans. Amer. Math. Soc. 353 (2001) 3649-3659.

[2] H. Berestycki, T. Cazenave, Instabilité des états stationnaires dans les équations de Schrödinger et de Klein-Gordon non linéaires, C. R. Acad. Sci. Paris 293 (1981) 489-492.

[3] H. Brézis, E.H. Lieb, A relation between pointwise convergence of functions and convergence of functionals, Proc. Amer. Math. Soc. 88 (1983) 486-490.

[4] T. Cazenave, Semilinear Schrödinger equations, Courant Lecture Notes Math., vol. 10, New York University, Courant Institute of Mathematical Sciences, New York, American Mathematical Society, Providence, RI, 2003.

[5] T. Cazenave, P.L. Lions, Orbital stability of standing waves for some nonlinear Schrödinger equations, Comm. Math. Phys. 85 (1982) 549-561.

[6] J. Fröhlich, E.H. Lieb, M. Loss, Stability of Coulomb systems with magnetic fields I. The one-electron atom, Comm. Math. Phys. 104 (1986) 251-270.

[7] M. Grillakis, J. Shatah, W. Strauss, Stability theory of solitary waves in the presence of symmetry, I, J. Funct. Anal. 74 (1987) $160-197$.

[8] M. Grillakis, J. Shatah, W. Strauss, Stability theory of solitary waves in the presence of symmetry, II, J. Funct. Anal. 94 (1990) $308-348$.

[9] Guo Boling, Wu Yaping, Orbital stability of solitary waves for the nonlinear derivative Schrödinger equation, J. Differential Equations 123 (1995) 35-55.

[10] N. Hayashi, The initial value problem for the derivative nonlinear Schrödinger equation in the energy space, Nonlinear Anal. 20 (1993) 823-833.

[11] N. Hayashi, T. Ozawa, On the derivative nonlinear Schrödinger equation, Physica D 55 (1992) 14-36.

[12] N. Hayashi, T. Ozawa, Finite energy solutions of nonlinear Schrödinger equations of derivative type, SIAM J. Math. Anal. 25 (1994) 14881503.

[13] E.H. Lieb, On the lowest eigenvalue of the Laplacian for the intersection of two domains, Invent. Math. 74 (1983) $441-448$.

[14] W. Mio, T. Ogino, K. Minami, S. Takeda, Modified nonlinear Schrödinger equation for Alfvén waves propagating along the magnetic field in cold plasmas, J. Phys. Soc. Japan 41 (1976) 265-271.

[15] E. Mjølhus, On the modulational instability of hydromagnetic waves parallel to the magnetic field, J. Plasma Phys. 16 (1976) $321-334$.

[16] H. Nawa, Asymptotic profiles of blow-up solutions of the nonlinear Schrödinger equation with critical power nonlinearity, J. Math. Soc. Japan 46 (1994) 557-586.

[17] M. Ohta, Stability of standing waves for the generalized Davey-Stewartson system, J. Dynam. Differential Equations 6 (1994) 325-334. 
[18] M. Ohta, Stability and instability of standing waves for one-dimensional nonlinear Schrödinger equations with double power nonlinearity, Kodai Math. J. 18 (1995) 68-74.

[19] M. Ohta, Stability and instability of standing waves for the generalized Davey-Stewartson system, Differential Integral Equations 8 (1995) $1775-1788$.

[20] M. Ohta, Blow-up solutions and strong instability of standing waves for the generalized Davey-Stewartson system in $\mathbb{R}^{2}$, Ann. Inst. H. Poincaré Phys. Théor. 63 (1995) 111-117.

[21] T. Ozawa, On the nonlinear Schrödinger equations of derivative type, Indiana Univ. Math. J. 45 (1996) 137-163.

[22] J. Shatah, Stable standing waves of nonlinear Klein-Gordon equations, Comm. Math. Phys. 91 (1983) 313-327.

[23] J. Shatah, W. Strauss, Instability of nonlinear bound states, Comm. Math. Phys. 100 (1985) 173-190.

[24] H. Takaoka, Well-posedness for the one-dimensional nonlinear Schrödinger equation with the derivative nonlinearity, Adv. Differential Equations 4 (1999) 561-580.

[25] H. Takaoka, Global well-posedness for Schrödinger equations with derivative in a nonlinear term and data in low-order Sobolev spaces, Electron. J. Differential Equations 2001 (42) (2001) 1-23.

[26] M.I. Weinstein, Nonlinear Schrödinger equations and sharp interpolation estimates, Comm. Math. Phys. 87 (1983) $567-576$.

[27] M.I. Weinstein, Lyapunov stability of ground states of nonlinear dispersive evolution equations, Comm. Pure Appl. Math. 39 (1986) 51-68. 